\title{
Cardiac arrest during laparoscopic cholecystectomy under general anaesthesia: A study into four cases
}

\section{Gautam B ${ }^{1}$, Shrestha BR ${ }^{2}$}

${ }^{1}$ Lecturer, ${ }^{2}$ Associate Professor, Department of Anaesthesiology and Intensive Care, Kathmandu Medical College, Sinamangal, Nepal

\begin{abstract}
Laparoscopic cholecystectomy (LapChole) has virtually superseded the more conventional open abdomen approach for the surgical treatment of symptomatic cholelithiasis. LapChole is however not a risk free procedure and serious, potentially fatal intra-operative complications can occur. Here we present case reports of four patients who suffered from intra-operative cardiac arrest during LapChole. All four recovered without residual morbidity and three of them underwent successful surgery in the same setting. No definite cause could be identified in any of the patients. We outline several possible mechanisms that could have been involved and discuss these events in face of published reports describing similar incidences. We infer that the creation of carbon-dioxide $\left(\mathrm{CO}_{2}\right)$ pneumoperitoneum was involved in the causation of the cardiac arrest because all four incidences occurred within minutes thereafter. Although rare, such complications can be fatal and are thus demanding to the anaesthesiologist.
\end{abstract}

Key words: Anaesthetic complications; carbon-dioxide pneumoperitoneum (CP); cardiac arrest; general anaesthesia; laparoscopic cholecystectomy

\begin{abstract}
Soon after its introduction in the 1950 s by the gynaecologists as a technique to diagnose pelvic pain, laparoscopy established itself as a surgical technique. The emergence of laparoscopic surgery has changed the way several organs are approached on the operation table. In the treatment of diseases like symptomatic cholelithiasis the laparoscopic approach has virtually replaced the open abdomen approach, with the latter being employed only if the former fails. The laparoscopic approach boasts several advantages over the conventional approach. These include reduction of postoperative pain, better cosmetic results, quicker return to normal activities, reduction in hospital stay resulting in overall reduction in total cost of treatment, less intra-operative bleeding, less postoperative wound infection, reduced metabolic derangement and better postoperative pulmonary function ${ }^{1,2,3}$. The success in healthy adult patients has led to the introduction of more extensive laparoscopic procedures in older and sick patients, as well as in pregnant and paediatric patients.
\end{abstract}

Laparoscopic procedures attempt to achieve minimal invasion of body tissues. Nevertheless they are not benign risk-free surgeries and are often associated with intra-operative haemodynamics derangements. An iatrogenic increment of pressure within a closed abdominal cavity during LapChole poses a definite challenge to the containing organs and vessels. The resulting variable impedance to the normal flow of blood brings the body's compensatory mechanisms into play in the form of metabolic, endocrine and autonomic nervous system responses. Patient's ability to tolerate and to respond to the various stresses is widely variable because the factors likely to influence the intra-operative cardiovascular function are numerous ${ }^{4-8}$ during LapChole performed under general anaesthesia. The interaction of physiology with the iatrogenic stresses may manifest intra-operatively as an alteration in electrical and mechanical activity of the heart together with alterations in measured blood pressure. The haemodynamics changes, which are principally attributable to raised intra-abdominal pressure (IAP) due to $\mathrm{CP}$ and patient's positioning on the operating table $4,5,6,7,8$, obviously get exaggerated in elderly and sicker patients ${ }^{9,10}$.

In addition, numerous procedure-related intra-operative complications of LapChole have been reported ${ }^{1,8,11,12,13}$. Several of the physiological changes and the procedurerelated complications can take a serious course and if not

Correspondence

Dr. Binod Gautam

Lecturer, Department of Anaesthesiology and Intensive Care, Kathmandu Medical College Teaching Hospital,

Sinamangal, Kathmandu, Nepal

E-mail: gautambinod@hotmail.com 
attended with prompt and appropriate intervention may terminate in to cardiac arrest, increasing the possibility of permanent injury or demise of the patient. Although adverse incidents are not common, laparoscopic surgery is a demanding procedure for the anaesthesiologist involved. Realization of the seriousness of such problems might provide the impetus to work towards the development of alternative anaesthetic regimen that minimises the risk of such deadly complications.

Here we report on four anaesthetised patients who went through intra-operative cardiac arrest after the creation of CP during LapChole.

\section{Patient 1}

A 58 year old woman, suffering from symptomatic cholelithiasis, was admitted for LapChole. She had a medical history of non insulin dependent diabetes mellitus treated with Metformin for the last 6 years. She had no drug allergies and was a non-smoker. Physical examination was unremarkable; weight was $62 \mathrm{~kg}$, and height $162 \mathrm{~cm}$. Preoperative fasting blood sugar, serum electrolytes, urea and creatinine were all within normal limits. She had a normal chest X-ray and the electrocardiogram (ECG) done 16 hours before surgery showed a normal sinus rhythm with a heart rate (HR) of $92 / \mathrm{min}$.

In the operating room (OR), standard monitors (ECG, pulse oximetry, non invasive blood pressure-'NIBP') wereplaced andanaesthesiawas induced withMeperidine $60 \mathrm{mg}$ and Thiopentone $300 \mathrm{mg}$ intravenously (IV). Succinylcholine $100 \mathrm{mg}$ IV was given to facilitate orotracheal intubation. After uneventful tracheal intubation, capnography was instituted to monitor end tidal $\mathrm{CO}_{2}$ values and Vecuronium $3 \mathrm{mg}$ IV was used for muscle relaxation. The ventilator was initially set at a tidal volume of $600 \mathrm{ml}$, frequency of $12 / \mathrm{min}$ at a fresh gas flow rate of $5 \mathrm{~L} / \mathrm{min}$ employing circle breathing circuit. After she received $10 \mathrm{mg}$ Metoclopramide IV, an orogastric tube was placed and suction was applied to decompress the stomach. The patient was prepared, draped, and each anticipated port site was infiltrated with $3 \mathrm{ml}$ of $0.25 \%$ Bupivacaine by the surgeon. Anaesthesia was maintained with $0.5-1.5 \%$ of Halothane in Oxygen.

The first (umbilical) port was created and through which the abdomen was insufflated with $\mathrm{CO}_{2}$ at a flow rate of $1 \mathrm{~L} / \mathrm{min}$ and IAP was maintained below $15 \mathrm{mmHg}$ throughout. Two other ports were created through which surgeons started dissecting the gall bladder, when sudden bradycardia was noticed. This rapidly progressed to asystole before administering $0.6 \mathrm{mg}$ Atropine and ECG, NIBP and pulse oximetry signals became unrecordable. End tidal $\mathrm{CO}_{2}$ decreased from $35 \mathrm{mmHg}$ to $28 \mathrm{mmHg}$. The surgeons were notified.
They immediately desufflated the abdomen and chest compressions were started. Normal sinus rhythm appeared at approximately 30 seconds after initiating the chest compressions, with a HR of $58 / \mathrm{min}$ together with a NIBP of $110 / 86 \mathrm{mmHg}, \mathrm{SPO}_{2}$ of $98 \%$ and end tidal $\mathrm{CO}_{2}$ of $32 \mathrm{mmHg}$. Stable haemodynamics persisted for another $10 \mathrm{~min}$ and the surgeons started the surgical procedure again. There was no deviation from the normal haemodynamics and LapChole was completed without difficulty within the next 40 minutes. At the end of the procedure, neuromuscular blockade was reversed with Neostigmine and Atropine, and the trachea was extubated while the patient was awake in the OR prior to transfer to the intensive care unit.

In the search for any precipitating cause several investigations were performed. Repeated cardiac enzymes and ECGs were all described as normal, as was an echocardiogram. This patient had well controlled blood sugar and serum electrolyte levels. Importantly she made an uneventful recovery.

\section{Patient 2}

A 38 year old woman with chronic calculus cholecystitis appeared for LapChole. She had a medical history of one episode of infectious hepatitis 8 years back. She was a non-smoker, non-drinker, had no known allergies, and had undergone caesarean section under uneventful subarachnoid block 12 years back. Physical examination was unremarkable, with a body weight of $57 \mathrm{~kg}$ and height of $158 \mathrm{~cm}$. Preoperative laboratory tests were all within normal limits.

She appeared in the OR at 1:00 pm. Routine monitors were applied. Her preoperative NIBP and HR were 100/66 $\mathrm{mmHg}$ and 106/min respectively. Anaesthesia was induced with Meperidine $55 \mathrm{mg}$ and Thiopentone $275 \mathrm{mg}$ IV. Succinylcholine $100 \mathrm{mg}$ IV was employed to facilitate intubation. Anaesthesia was maintained with $0.5-1.5 \%$ Halothane in Oxygen. Vecuronium 3 $\mathrm{mg}$ was administered to maintain muscle relaxation. $\mathrm{CP}$ was created and IAP was maintained at $15 \mathrm{mmHg}$. NIBP recorded while the surgeon was preparing to make the third port site revealed a drop to $82 / 54$ $\mathrm{mmHg}$. Mephenteramine $12 \mathrm{mg}$ IV was administered. Immediately afterwards, the patient developed pulseless electrical activity (PEA) with the pulse oximeter waveform and the arterial blood pressure becoming unobtainable. The end tidal $\mathrm{CO}_{2}$ concentration decreased from $33 \mathrm{~mm} \mathrm{Hg}$ to $16 \mathrm{~mm} \mathrm{Hg}$. ECG architecture was looking normal with a HR of $92 / \mathrm{min}$. An Advanced Cardiopulmonary Life Support protocol for PEA was immediately initiated which included chest compressions and IV doses of Epinephrine 1 $\mathrm{mg}$, two doses approximately $3 \mathrm{~min}$ apart. Halothane administration was immediately discontinued and the 
surgeons were asked to desufflate the abdomen. The patient had received $350 \mathrm{ml}$ of normal saline till then. She was infused with another $700 \mathrm{ml}$ normal saline within 5 minutes. After 3-4 min of initiation of the chest compressions, normal peripheral circulation with a pulse rate of 112/min and NIBP of 117/63 mm Hg were achieved. The operative procedure was recommenced in the usual fashion once her hemodynamic parameters stabilized without requiring pharmacological support. Her calculous gall bladder was successfully removed within an eventless operation lasting $34 \mathrm{~min}$.

Once the patient was awake and responding to verbal command as well as upon return of adequate neuromuscular function, the trachea was extubated in the OR. She was then transported to the intensive care unit and had no postoperative troubles referable to her intra-operative cardiac arrest.

\section{Patient 3}

A 64 year old woman with chronic calculus cholecystitis was scheduled for LapChole. She had been on regular oral Atenolol for her hypertension since the last eight years. She did not have any history of chest pain, syncope, and dyspnea and had a good exercise tolerance. She was a non-smoker, non-drinker, had no known allergies, and had had no previous anaesthetic exposure. Physical examination performed a day prior to surgery revealed a HR of 66/min and a blood pressure of 150/92 $\mathrm{mmHg}$ in both arms. Jugular veins were not distended and her chest was clear on auscultation. She weighed $65 \mathrm{~kg}$ and had a height of $164 \mathrm{~cm}$. Preoperative ECG and chest X-ray and other laboratory tests were within normal limits. She received $5 \mathrm{mg}$ Diazepam orally the night prior to surgery. She also received $5 \mathrm{mg}$ Diazepam and $25 \mathrm{mg}$ Atenolol orally at 8:00 am on the morning of surgery.

In the OR she seemed to be anxious and had a HR of $82 / \mathrm{min}$ and a NIBP of 156/96 mmHg. With Midazolam $3 \mathrm{mg} \mathrm{IV}$, she became calm and HR came down to 68/ min and NIBP became 144/90 mmHg. Anaesthesia was then induced with Meperidine $65 \mathrm{mg}$, Lidocaine 100 $\mathrm{mg}$, Thiopentone $325 \mathrm{mg}$ and Vecuronium $8 \mathrm{mg}$. While awaiting deep anaesthesia and attenuation of laryngopharyngeal reflexes, positive pressure ventilation was undertaken with $1.5 \%$ of Halothane in Oxygen. After two minutes, her HR and NIBP read 106/min and $166 / 100 \mathrm{mmHg}$ respectively. Labetalol $10 \mathrm{mg}$ was administered and anaesthesia was further deepened with $2 \%$ Halothane for another 2 minutes. With this, she was well relaxed with a HR of $64 / \mathrm{min}$ and a NIBP of 124/78 $\mathrm{mmHg}$. Laryngoscopy and intubation were performed without difficulty within 10 seconds, but her NIBP in-between increased up to $168 / 104 \mathrm{mmHg}$ and the HR reached up to $96 / \mathrm{min}$ with occasional ventricular premature contractions. Labetalol $5 \mathrm{mg}$ was injected twice within a period of the next five minutes. She was ventilated with $2.5 \%$ of Halothane for another 2 minutes when her heart rate and NIBP were recorded at $68 / \mathrm{min}$ and $132 / 70 \mathrm{mmHg}$ respectively. Anaesthesia was then maintained with $1.5 \%$ Halothane in Oxygen which maintained NIBP at around 130-140/64-92 $\mathrm{mmHg}$ and HR between 56-70/min.

$\mathrm{CP}$ was being created with $\mathrm{CO}_{2}$ administration at a flow rate of $1 \mathrm{~L} / \mathrm{min}$ when sudden asystole was noted on the continuous ECG. This was concomitant with the loss of the pulse oximetry and capnography signals during the later period of creation of CP and she had no radial and carotid arterial pulses. Chest compressions were initiated, Atropine $(0.6 \mathrm{mg}$ IV) was administered and $\mathrm{CO}_{2}$ insufflation and Halothane administration were interrupted immediately and the abdomen was desufflated. After approximately two minutes of chest compressions and another $0.6 \mathrm{mg}$ Atropine administration, normal sinus rhythm was restored. The operative procedure was cancelled as the patient was then ventilated with $100 \%$ Oxygen at a rate of $12 / \mathrm{min}$ with a tidal volume of $550 \mathrm{ml}$. The patient emerged from anaesthesia without difficulty and was drowsy but easily arousable in the first $20 \mathrm{~min}$ after the event. Neuromuscular blockade was reversed pharmacologically once she demonstrated spontaneous breathing effort after 35 minutes of the event. The trachea was extubated with the patient awake in the OR.

Immediate postoperative ECG revealed new onset T-wave inversion on the lateral chest leads. She was admitted to the intensive care unit and subsequent serial ECGs and cardiac enzymes ruled out a myocardial infarction. Echocardiography performed 24 hours later was unremarkable except mild diastolic dysfunction and an ejection fraction of $48 \%$. Her blood pressure remained normal with Atenolol and she made an uneventful recovery.

\section{Patient 4}

A 27 year lady weighing $56 \mathrm{~kg}$ with an ASA physical status I and the diagnosis of multiple calculus cholelithiasis was posted for LapChole. She didn't have any notable findings in history, clinical examination and basic investigations. Gabapentin $600 \mathrm{mg}$ orally was employed for premedication one hour prior to surgery.

Upon arrival in OR at 9:15 am she had a HR of 72/ min in a normal sinus rhythm, NIBP 120/70 mmHg and $\mathrm{SPO}_{2}$ of $98 \%$ without supplemental oxygen. Ceftriaxone $1 \mathrm{gm}$ IV was administered slowly after securing an IV access. Anaesthesia was induced with Fentanyl 100 $\mathrm{mcg}$, Propofol $170 \mathrm{mg}$ and Vecuronium $5.5 \mathrm{mg}$ and 
positive pressure ventilation was carried out with $1.5 \%$ Halothane in Oxygen. After 3 minutes, when she had her HR of $62 / \mathrm{min}$ and NIBP of $115 / 85 \mathrm{mmHg}$, laryngoscopy and intubation were done. Her subsequent HR and NIBP recorded at $85 / \mathrm{min}$ and $170 / 113 \mathrm{mmHg}$ respectively. Despite increasing Halothane concentration to $2 \%$ her NIBP did not drop down below 160/110 mm Hg. Labetalol $10 \mathrm{mg}$ was administered in increments and titrated finally to the HR of $60 / \mathrm{min}$ and NIBP of $130 / 80$ $\mathrm{mmHg}$ within 6 minutes. Anaesthesia was maintained with 1\% Halothane in Oxygen with NIBP 95/60 mm Hg and HR $62 / \mathrm{min}$. CP was created at a flow rate of $1 \mathrm{~L} / \mathrm{min}$ and IAP fixed at $14 \mathrm{mmHg}$. The patient was ventilated manually and end tidal $\mathrm{CO}_{2}$ recorded was in the range of $32-35 \mathrm{mmHg}$.

Fourteen minutes after induction of anaesthesia, when surgeons were dissecting on the gall bladder bed, HR suddenly dropped to $20 / \mathrm{min}$. Halothane was immediately switched off, fluid was infused at its maximum rate and surgeons were made aware of the incident. ECG spontaneously and rapidly decayed in to isoelectric before administering Atropine $1.2 \mathrm{mg}$ IV and prior to deflation of CP. Pulse was not palpable and NIBP recorded at 40/12 $\mathrm{mm} \mathrm{Hg}$. Upon immediate Atropine administration, deflation of abdomen and resuming supine position, ECG returned to sinus rhythm with HR of $47 / \mathrm{min}$, which gradually increased and then settled at around 109/min and so did her NIBP at 90105/50-65 mmHg. CP was created again and LapChole was accomplished within next 15 minutes without any significant alteration in haemodynamics. Trachea was extubated following reversal of the effect of muscle relaxant with Atropine $1.2 \mathrm{mg}$ followed 2 minutes later by Neostigmine $2.5 \mathrm{mg}$. Her post operative recovery was smooth.

\section{Discussion}

In a period of five years from July 1, 2004 to July 2009, 2217 patients were operated for cholecystectomy in Kathmandu Medical College-Teaching Hospital. 72 of the gall bladders were removed by the conventional open abdomen surgical approach whereas 2145 were approached laparoscopically. LapChole has overtaken open abdomen surgery as the preferred approach to cholecystectomy ${ }^{3}$. Although it was initially used exclusively in healthy and young patients, in the face of improving technology and growing surgical expertise, more extensive and prolonged laparoscopic procedures are proving to be successful and safe in a wide range of patients. Despite several advantages like less pain, faster recovery and possibly less morbidity and mortality, laparoscopic surgeries are not risk free and the importance of caution, observation and prudent intervention in the part of the team involved in the operation cannot be exaggerated.
We reported here on four patients who suffered non-fatal cardiac arrest after the creation of CP during LapChole. Such incidences are not very common- we encountered these cases in the course of five years during which we were involved in performing 2145 LapCholes. However, given the seriousness of the problem and the possible clinical progress these are significant issues and therefore we deem it important to carefully study and register such events. The wide variability in the incidence and outcomes of cardiac arrest ${ }^{14,15,16 \text {, }}$ underlines the need for individual hospitals to know its numbers. Among others, poor reporting of the perioperative critical events seems to be the principal reason behind scarcity of data in Nepalese literature.

The creation and maintenance of $\mathrm{CP}$ at a raised IAP, a defining step during LapChole, has got the inherent potential for causing morbidity not only in ASA III-IV patients ${ }^{9,10}$ but also in healthy ASA I-II patients, 5, 6, 7, ${ }_{\text {. }}$. Other factors like the rate and duration of administration of $\mathrm{CO}_{2}$, the patient's age, underlying cardiovascular function, pre-existent co-morbidities ${ }^{7,8,9,10,11,12}$, intravascular volume status ${ }^{17,18}$, and positioning on the operation table, the ventilatory technique, the surgical conditions, and the choice of anaesthetic technique and agents can also contribute to the intra-operative haemodynamics destabilisation.

Hemodynamic alterations associated with LapChole include but are not limited to an increase in mean arterial pressure, systemic and pulmonary vascular resistance, a decrease in ejection fraction ${ }^{6}$ and a decrease in cardiac index ${ }^{7}$. The head-up position employed during LapChole causes a reduction in venous return and cardiac output, as well as an increase in peripheral and pulmonary vascular resistance ${ }^{19,20}$ consequently increasing the left ventricular end-systolic wall stress ${ }^{6}$. Cardiac output often increases initially due to splanchnic compression but within minutes, it decreases due to reduced venous return and decreased myocardial contractility. This is further aggravated by an increase in afterload and systemic vascular resistance ${ }^{21}$. Patients with good general condition and normal cardiovascular function are able to tolerate these variations well but those with cardiovascular disease, anaemia, or hypovolemia require meticulous consideration to volume loading, positioning, and insufflation pressures in order to avoid serious cardiac events.

Many cases of cardiovascular collapse during laparoscopic procedures, however, occur in healthy patients, most notably due to profound vagal response to peritoneal stimulation, myocardial sensitisation by Halothane, reduced venous return secondary to reverse Trendelenburg position, compression of inferior vena-cava, acute blood loss and anaesthetic agents. 
In addition, high insufflation pressures, hypovolemia, venous gas embolism, myocardial dysfunction, myocardial ischemia, cardiac dysrhythmias, tension pneumothorax, pneumomediastinum, subcutaneous emphysema, profound hypercarbia and interplay of potentially lethal anaesthetic medications have also been implicated ${ }^{1,8,12,13}$. Advanced age, in itself, may not be a risk factor for intra-operative complications but the underlying co-morbidities that develop as part of the aging process increases the risk of peri-operative cardiac arrest ${ }^{16,22}$.

Procedure-related intra-operative complications during LapChole including puncture of hollow viscus and major vessels, haemorrhage, pneumomediastinum, pneumothorax, subcutaneous emphysema and cardiac tamponade were excluded as the primary reason for causing cardiac arrest in our patients, as concluded from our clinical observations. Although clinically apparent $\mathrm{CO}_{2}$ embolism is a rare complication, the incidence of subclinical embolic events is not uncommon during LapChole, especially during peritoneal insufflation and gall bladder dissection ${ }^{12}$. We suspect that rather than a massive gas embolism being a primary reason for the genesis of cardiac arrests in our patients, subclinical gas embolism might have played some contributory role.

Cardiac arrest in all of these patients occurred within minutes of creation of $\mathrm{CP}$ which reflects that hypercarbia was not certainly the causative factor; this was confirmed by clinical judgment and immediate pre-arrest end tidal $\mathrm{CO}_{2}$ values. Thus, we infer that the increased IAP and the maintenance of CP must have been the principal factor, compounded with others, in the causation of cardiac arrest in our patients.

Patient 1 did not have any predisposing cardiac history nor did she receive any cardiac medications prior to the episode of asystole. The only potential predisposing factor was diabetes mellitus. Diabetes is associated with autonomic neuropathy and cardiovascular involvement ${ }^{23}$. A peak-basal heart rate ratio of less than 1.2 on Dipyridamole myocardial single photon emission computed tomography (SPECT) has been advocated as an indicator of cardiac autonomic dysfunction in diabetics ${ }^{24}$. Our patient did not undergo standardized diagnostic tests for diabetic autonomic neuropathy or any formal assessment of her heart rate variability. Still, in the absence of other tests, the possibility of autonomic neuropathy cannot be completely excluded. Diabetics are at increased risk for cardiovascular lability during anaesthesia ${ }^{25}$ and preoperative screening of diabetics with simple non invasive autonomic tests may be useful in identifying those at high risk for peri-operative hemodynamic instability.
Although standard pharmacology textbooks do not mention Metoclopramide producing cardiovascular side effects, several cases of associated bradycardia or complete heart block and hypotension within minutes of IV Metoclopramide administration have been reported in various clinical settings ${ }^{26,27}$. Malkoff et $a l^{28}$ reported a case of sinus arrest after IV Metoclopramide and suggested that autonomic dysfunction could be a risk factor for Metoclopramide-associated arrhythmias. However, Metoclopramide has been also shown to predispose sinus arrest in diabetics even without overt autonomic neuropathy ${ }^{29}$. It seems that Metoclopramide, used for its prokinetic action affected by the release of acetylcholine in gastrointestinal tract, is likely to disturb cardiovascular system at least in predisposed patients. The mechanisms underlying potential arrhythmogenic effects of IV Metoclopramide are not completely understood, but its structural similarities to procainamide ${ }^{30}$ might play a role.

IV administration of Metoclopramide in the presence of diabetic autonomic neuropathy could have been the most likely culprit in the precipitation of bradycardia progressing to asystole in Patient 1. In addition, reflex vagal hyperactivity due to peritoneal stretching and gall bladder dissection and the use of drugs like Halothane and Vecuronium could have been contributory.

PEA occurring during general anaesthesia could be explained by various mechanisms and hypovolemia is the most important one. Incompletely treated hypovolemia could not be excluded in Patient 2 . She had had her last oral intake almost $13 \mathrm{hrs}$ back and her calculated fasting fluid deficit was $1261 \mathrm{ml}$ whereas she had received only $350 \mathrm{ml}$ of IV fluid before the event. Moreover, aortocaval compression due to increased IAP might have aggravated the pre-existent hypovolemia to the extreme effect of almost depriving the heart of any blood return. Patients who experience the most severe hemodynamic changes during $\mathrm{CP}$ are said to have a low preoperative cardiac output and central venous pressure and a high mean arterial pressure and systemic vascular resistance - a profile suggesting depleted intravascular volume ${ }^{10}$.

The combination of raised IAP, pre-existent hypovolemia and Halothane anaesthesia is the most likely explanation for the causation of PEA in our otherwise healthy Patient 3 . A recorded arterial pressure of 82/54 mmHg just before the event and rapid return of cardiac pumping activity with chest compressions, release of $\mathrm{CP}$, rapid fluid infusion and administration of vasopressor further support our assumption. The prognosis of PEA in an anaesthetized patient is grim unless resuscitation involves finding and treating the 
precipitating cause. In addition to routine evaluation of depth of anaesthesia and intravascular volume status, it must be confirmed that the IAP has not exceeded 15 $\mathrm{mmHg}$ during LapChole. Recent studies recommend a moderate to low IAP $(<12 \mathrm{mmHg})$ as it limits the alteration in splanchnic perfusion, and consecutive organ dysfunctions will be minimal, transient, and will not influence the outcome ${ }^{19,31}$.

Patient 3 was hypertensive for eight years and was on Atenolol since then but her immediate preoperative NIBP was $156 / 96 \mathrm{mmHg}$. Safrin et $a l^{10}$ had pointed out that patients having high preoperative values of mean arterial pressure and systemic vascular resistance are more likely to experience the most severe hemodynamic changes during laparoscopy. Although a definitive cause for asystole in this patient could not be determined, there is much circumstantial evidence for multipledrug toxicities leading to sinoatrial node failure and asystole. The predominant mechanism in medicationrelated cardiac arrests in various studies is found to be cardiovascular depression which in our Patient 3 holds true; it was not the use of incorrect drug, but rather a relative overdose administered or an unusual response to a standard dose. She received a combination of many cardio-suppressant drugs. First of all, relative overdose of or exaggerated response to beta-antagonist drugs cannot be excluded. She received $25 \mathrm{mg}$ Atenolol orally one hour before induction of anaesthesia and $20 \mathrm{mg}$ Labetalol in divided doses before the event occurred. Labetalol is used extensively for controlling blood pressure in the peri-operative period. Reductions in ventricular contractility from beta-adrenergic blocking effects of Labetalol and limited vasoconstrictive compensatory mechanisms due to its alpha-adrenergic blocking effects might prove hazardous leading to a state of cardiovascular collapse ${ }^{32}$. Furthermore, vagal reflex is accentuated if the patient is taking beta-blocking drugs $^{33}$. Secondly, deepening of anaesthesia with up to $2.5 \%$ of Halothane for almost six minutes might have been implicated ${ }^{17,34}$. Moreover, the intubating dose of Vecuronium in combination with the above mentioned drugs together with the inherent capability of raised IAP and CP precipitating vagal reflex was the most likely explanation of asystole in our Patient 3.

The phenomenon of vagus-mediated reflex bradycardia and sinus arrest has long been observed during a variety of surgical stimuli ${ }^{35}$ including laparoscopic surgeries ${ }^{36,37}$. Significant bradycardia, atrioventricular dissociation, and asystole have been reported during LapChole which are attributed to vagal stimulation caused by insertion of the Veress needle or the trocar, CP-induced peritoneal stretch, or $\mathrm{CO}_{2}$ embolization $^{38}$. Many anaesthetic drugs such as Vecuronium, Halothane, and Succinylcholine which lack vagolytic effects may allow drug or reflex-induced bradycardia and asystole to occur more easily ${ }^{35}$. Vecuronium, a non-depolarizing neuromuscular blocking agent, is well known for its cardiovascular stability and has been shown to facilitate vagal reflex and to precipitate severe bradycardia and asystole ${ }^{36,37,39}$. Halothane causes bradycardia by interfering with calcium channels, resulting in a reduction in contractility and a slowing of conduction ${ }^{34}$. Further, bradycardia caused by Halothane may be exaggerated leading to severe bradycardia and asystole in patients receiving high doses of Vecuronium and/or Beta-antagonists ${ }^{33}$.

The stress response, against laryngoscopy and intubation, CP, general anaesthesia with positive pressure ventilation and the surgery itself, continues to be a feared phenomenon during the peri-operative periods of LapChole. Various agents and anaesthetic techniques have been employed to minimise the deleterious effects of stress response with varied efficacy. Not all patients have to respond to stress in one and the similar way as we often expect. Some of our patients may exhibit their autonomic deviation, often difficult to foresee, with parasympathetic dominance. On top of that the hemodynamic effects of CP compounded by the concomitant use of Fentanyl ${ }^{40}$, Propofol ${ }^{41}$, Halothane, Vecuronium and Labetalol must have worked in an additive fashion $^{42}$ in our Patient 4 to exacerbate bradycardia terminating in asystole. The time of peak effect of Labetalol ${ }^{43}$ in this patient coincided with the moment of the event noticed.

Although hypoxemia is classically considered the most frequent cause of anaesthesia-related cardiac $\operatorname{arrest}^{14,15}$, it was certainly not the factor in our patients. Human error has long been identified as a major factor leading to anaesthesia-related cardiac arrest, with poor preoperative preparation being recognised as the most often identified factor ${ }^{14,15,16,44}$. Since all identified errors do not ultimately terminate in a cardiac arrest, it's evident that the incidence of errors must be far greater than that observed in many studies. Human error cannot be completely denied in our patients too. Failure to anticipate and recognize autonomic neuropathy in Patient 1 can be considered as an error. Similarly, lack of recognition and inadequate treatment of hypovolemia in Patient 2 is definitely a human error which in the presence of surgically created CP ultimately precipitated $P E A$. Inadequate or inappropriate risk estimation pre-operatively and misjudgements occurring intra-operatively seem to be the major human errors responsible for triggering the chain of crisis.

Preventing human error, to a large extent, is a complex task. Continued medical education is fundamental but not sufficient to prevent critical incidents occurring 
during anaesthesia. Lack of situation awareness rather than a lack of acquired knowledge is an important and growing concern. It is the poor practical appliance of knowledge that leads to critical incidents ${ }^{45}$. In anaesthesia, research based on models derived from aeronautics has started ${ }^{46,47}$ and training programs based on simulations of critical anaesthetic scenarios have been proposed and in use for improving anaesthetic safety $^{48}$. Today, anaesthesia is usually a concomitant and not a causative factor for peri-operative cardiac arrest. Vast majority of cardiac arrests during general anaesthesia must be ascribed to patient's pre-existing status, especially the cardiovascular system's interplay with autonomic nervous system. In addition, surgical factors are shown to be the important source of fatal outcome ${ }^{49}$.

In conclusion, the cardiac arrest witnessed during LapChole performed under general anaesthesia is multi-factorial in origin, associated with the nature of the surgical technique, poor preoperative patient condition, inadequate preoperative risk estimation, and intra-operative errors or misjudgements. We have pointed out that LapChole is not a trivial procedure because it has got the potential to kill otherwise healthy persons and our experience confirms that a good result can often be obtained provided cardiac arrest is quickly spotted and treated. One needs to be aware of the possible alterations and complications during laparoscopic surgeries and must be well rehearsed about what to do should the crisis occur. The caring team should realize the importance of being vigilant and consider employing appropriate monitoring techniques as well as ensuring the easy availability of emergency drugs and resuscitation equipments. Prompt diagnosis, timely decisions together with appropriate and adequate interventions without delay are the key to the successful resuscitation from intra-operative cardiac arrest.

Efforts should also be directed at making favourable OR working environment. In addition, behavioural and organisational factors should be addressed to prevent and control cardiac arrest. Gradual replacement of traditional inhalational agent (Halothane) with newer and less cardiac depressant agents definitely makes some difference. It has become by now high time to educate and convince the hospital management authority about such incidents because one will continue to face such problems unless we are made comfortable with the best available alternatives generated by science.

Cardiac arrest alarms death and terrifies all of those who witness it. In fact, the most dreaded event that could occur during surgery is the death of the patient on the operation table and it's the cardiac arrest that alarms its possibility. Some believe that the lower the incidence of intra-operative cardiac arrest, the more the competency and efficiency of the caring anaesthesiologist; while some might believe that the more the incidence of survival rate from the intra-operative cardiac arrest the more the efficiency of the caring system. But in reality both schools of thoughts can go wrong.

Anaesthesiology by nature involves crisis and if there were no incidences of peri-operative cardiac arrest, the speciality of anaesthesiology could not have advanced so much and also could not have gained such an importance and authority in recent years. As the prearrest morbidity scores have not yet been prospectively validated as instruments to predict the occurrence and survival rate after intra-operative cardiac arrest, it is so obvious that no one is immune to disaster during anaesthesia; neither the patient nor the anaesthesiologist. We must thus realize that cardiac arrest can happen to anyone and at any time despite our best attempts at preventing it; the motto is to be better prepared all the time throughout the peri-operative period to face and tackle it appropriately.

\section{References}

1. Wolfe BM, Gardiner B, Frey CF. Laparoscopic cholecystectomy. A remarkable development. JAMA. 1991; 265(12): 1573-4.

2. Patel N, Strogen CP, Kosinski R, Antiporda C. Yuvorek G, Baker T. The rapid recovery of respiratory function after laparoscopic cholecystectomy. Anesth Analg. 1991; 72: S 211.

3. Way LW. Changing therapy for gallstone disease. N Engl J Med. 1990; 323(18): 1273-4.

4. Marco AP, Yeo CJ, Rock P. Anaesthesia for a patient undergoing laparoscopic cholecystectomy. Anesthesiology. 1990; 73(6): 1268-70.

5. Critchley LA, Critchley JA, Gin T. Haemodynamic changes in patients undergoing laparoscopic cholecystectomy: measurement by transthoracic electrical bioimpedance. $\mathrm{Br} \mathrm{J}$ Anaesth. 1993; 70(6): 681-3.

6. Dorsay DA, Greene FL, Baysinger CL. Hemodynamic changes during laparoscopic cholecystectomymonitored withtransesophageal echocardiography. Surg Endosc. 1995; 9(2): 128-34.

7. Joris JL, Noirot DP, Legrand MJ, Jacquet NJ, Lamy ML. Hemodynamic changes during laparoscopic cholecystectomy. Anesth Analg. 1993; 76(5): 1067-71.

8. Hasel R, Arora SK, Hickey DR. Intraoperative complications of laparoscopic cholecystectomy. Can J Anaesth. 1993; 40(5 Pt 1): 459-64. 
9. Feig BW. Berger DH, Dupuis JF, et al. Hemodynamic effects of $\mathrm{CO}_{2}$ abdominal insufflation (CAI) during laparoscopy in highrisk patients. Anesth Analg. 1994; 78: SI 09.

10. Safran D, Sgambati S, Orlando R III. Laparoscopy in high-risk cardiac patients. Surg Gynecol Obstet. 1993; 176(6): 548-54.

11. Barone JE, Lincer RM. A prospective analysis of 1518 laparoscopic cholecystectomies. N Engl J Med. 1991; 324(16): 1073-8.

12. Derouin M, Couture P, Boudreault D, Girard D, Gravel D. Detection of gas embolism by transesophageal echocardiography during laparoscopic cholecystectomy. Anesth Analg. 1996; 82(1): 119-24.

13. Hall D, Goldstein A, Tynan E, Braunstein L. Profound hypercarbia late in the course of laparoscopic cholecystectomy: detection by continuous capnometry. Anesthesiology. 1993; 79(1): 173-4.

14. Olsson GL, Hallen B. Cardiac arrest during anaesthesia. A computer aided study in 250543 anaesthetics. Acta Anaesthesiol Scand. 1988; 32(8): 653-64.

15. Keenan RL, Boyan CP. Cardiac arrest due to anesthesia. A study of incidence and causes. JAMA. 1985; 253(16): 2373-7.

16. Newland MC, Ellis SJ, Lydiatt CA, Peters KR, Tinker JH, Romberger DJ, et al. Anestheticrelated cardiac arrest and its mortality: a report covering 72,959 anesthetics over 10 years from a US teaching hospital. Anesthesiology. 2002; 97(1): 108-15.

17. Diamant M, Benumof JL, Saidman LJ. Hemodynamics of increased intra-abdominal pressure: Interaction with hypovolemia and halothane anesthesia. Anesthesiology. 1978; 48(1): 23-7.

18. Ho HS, Saunders CJ, Corso FA, Wolfe BM. The effects of $\mathrm{CO}_{2}$ pneumoperitoneum on hemodynamics in hemorrhaged animals. Surgery. 1993; 114(2): 381-8.

19. Gutt CN, Oniu T, Mehrabi A, Schemmer P, Kashfi A, Kraus , et al. Circulatory and respiratory complications of carbon dioxide insufflation. Dig Surg. 2004; 21(2): 95-105.

20. Hirvonen EA, Poikolainen EO, Paakkonen ME, Nuutinen LS. The adverse hemodynamic effects of anesthesia, head-up tilt, and carbon dioxide pneumoperitoneum during laparoscopic cholecystectomy. Surg Endosc. 2000; 14(3): 272-7.

21. Kashtan J, Green JF, Parsons EQ, Holcroft JW. Hemodynamic effects of increased abdominal pressure. J Surg Res. 1981; 30(3): 249-55
22. Williams SL, Jones PB, PohalfWE. Preoperative management of the older patients-A surgeon's perspective: Part I. Clinical Geriatrics. 2006; 14:24-8.

23. Emanuele NV, Emanuele MA. Diabetic neuropathy: therapies for peripheral and autonomic symptoms. Geriatrics. 1997; 52(4): 40-9.

24. Lee KH, Yoon JK, Lee MG, Lee SH, Lee WR, Kim BT. Dipyridamole myocardial SPECT with low heart rate response indicates cardiac autonomic dysfunction in patients with diabetes. J Nucl Cardiol. 2001; 8(2): 129-35.

25. Burgos LG, Ebert TJ, Asiddao C, Turner LA, Pattison CZ, Wang-Cheng $\mathrm{R}$, et al. Increased Intraoperative Cardiovascular Morbidity in Diabetics with Autonomic Neuropathy. Anesthesiology. 1989; 70(4): 591-7.

26. Withington DE. Dysrhythmias following intravenous metoclopramide. Intensive Care Med. 1986; 12(5): 378-9.

27. Midttun M, Oberg B. Total heart block after intravenous metoclopramide (Letter). Lancet. 1994; 343(8890): 182-3.

28. Malkoff MD, Ponzillo JJ, Myles GL, Gomez CR, Cruz-Flores S. Sinus arrest after administration of intravenous metoclopramide. Ann Pharmacother. 1995; 29(4): 381-3.

29. Grenier Y, Drolet P. Asystolic cardiac arrest: an unusual reaction following iv metoclopramide. Can J Anaesth. 2003; 50(4): 333-5.

30. Ponte CD, Nappi JM. Review of a new gastrointestinal drug: metoclopramide. Am J Hosp Pharm. 1981; 38: 829-33.

31. Zuckerman RS, Heneghan S. The duration of hemodynamic depression during laparoscopic cholecystectomy. Surg Endosc. 2002; 16(8): 1233-6.

32. Tung A, Sweitzer BJ, Cutter T. Cardiac Arrest after Labetalol and Metoclopramide administration in a patient with scleroderma Anesth Analg. 2002; 95: 1667-8.

33. Joris JL. Anesthesia for Laparoscopic surgery. In: Miller RD (editors). Miller's Anesthesia. 6th edition. Philadelphia, Pennsylvania: Elsevier Inc.; 2005.p. 2293.

34. Maze A, Mason DM. Aetiology and treatment of halothane induced arrhythmias. Clinics in Anaesthesiology. 1983; 1: 301-21.

35. Doyle DJ, Mark PWS. Reflex bradycardia during surgery. Can J Anaesth. 1990; 37(2): 219-22.

36. Clayton D.Asystole associated with vecuronium. Br J Anaesth. 1986; 58(8): 937-8. 
37. Doyle DJ, Mark PWS. Laparoscopy and vagal arrest. Anaesthesia. 1989; 44(5): 448.

38. Sprung J, Abdelmalak B, Schoenwald PK. Recurrent complete heart block in a healthy patient during laparoscopic electrocauterization of the fallopian tube. Anesthesiology. 1998; 88(5): 1401-13.

39. Milligan KR, Beers HT. Vecuronium-associated cardiac arrest. Anaesthesia. 1985;40:385..

40. Royster RL, Keeler DK, Haisty WK, et al. Cardiac electrophysiologic effects of fentanyl and combinations of fentanyl and neuromuscular relaxants in pentobarbital anesthetized dogs. Anesth Analg. 1988; 67:15-20.

41. Cullen PM, Turtle M, Prys-Roberts C, et al: Effects of propofol anesthesia on baroreflex activity in human. Anesth Analg. 1987; 66:111520.

42. Kazuhiko Fukuda. Intravenous opioid anesthetics. In: Miller RD (editor). Miller's Anesthesia, 6th edition. Philadelphia, Pennsylvania: Elsevier Inc.; 2005.p. 394-95.
43. Sota Omoigui. Anesthesia drugs handbook. 3rd Ed. US: Blackwell Science, Inc.1999.

44. Pedersen T, Johansen SH. Serious morbidity attributable to anaesthesia. Considerations for prevention. Anaesthesia. 1989; 44(6): 504-8.

45. Sigurdsson GH, McAteer E. Morbidity and mortality associated with anaesthesia. Acta Anaesthesiol Scand. 1996; 40(8 Pt 2): 105763.

46. Arnstein F. Catalogue of human error. $\mathrm{Br} \mathrm{J}$ Anaesth. 1997; 79(5): 645-56.

47. Helmreich RL, Davies JM. Anaesthetic simulation and lessons to be learned from aviation (Editorial). Can J Anaesth. 1997; 44(9): 907-12.

48. Murray DJ, Boulet JR, Kras JF, J. A. Woodhouse, T. Cox and J. D. McAllister. Acute care skills in anesthesia practice: a simulation-based resident performance assessment. Anesthesiology. 2004; 101(5): 1084-95.

49. Zuercher M and Ummenhofer W. Cardiac arrest during anesthesia. Curr Opin Crit Care. 2008, 14: 269-74. 